\title{
The price of a kidney
}

T wo high-profile stories in US newspapers this year have prompted the media to ask whether the illegal sale of human organs in the US is more common than previously thought. In the first case, a New York City resident was arrested for trying to orchestrate the sale of a human kidney to an undercover FBI agent for US $\$ 160,000$. The second story was of an Israeli man who flew to New York to donate his kidney in exchange for $\$ 20,000$. These stories underscore the desperation of patients in need of a donor organ and the need to reduce the number of patients on transplant waiting lists. The methods to achieve this goal are severalfold and include increasing the number of live kidney donors, increasing deceased donation, and preventing the development of renal failure in the first instance.

Each day in the US, 17 people die waiting for a transplant. Although in the majority of countries, the selling of an organ is illegal, according to the WHO, $5-10 \%$ of all transplanted kidneys are purchased. The shortage of transplantable organs undoubtedly fuels the demand for illegally sourced organs. Critics of legislation in the US and most other countries argue that the legalization of kidney sales could increase donation and survival rates for patients on waiting lists. Several models of such regulation have been proposed-typically they involve the payment of a fixed sum to the donor, long-term follow-up and life insurance benefits. However, although a regulated system of organ sales could, in theory, enable follow-up and provision of adequate health care for kidney donors, this notion is at odds with the social and medical capabilities of many developing countries.

The argument that the regulated sale of organs could help lift the donor out of poverty has frequently been used; however, several studies have demonstrated that despite payment, donors often find themselves back in financial difficulty. Organs Watch has revealed that many paid donors suffer postoperatively from hypertension, chronic pain and social isolation. If their remaining kidney fails, many organ sellers do not have access to dialysis, let alone organ transplantation. As a result, several international organizations, including the WHO, condemn the sale of organs as financial incentives coerce organ donation, exploiting the vulnerable and the poor.

An alternative approach to increase the number of live donors is to reduce the disincentives associated with kidney donations, rather than by financially increasing the incentives, for example, by assuring donor safety and by safeguarding against loss of employment after donation. Such an approach would discourage international travel for financial gain and would best enable each country to provide transplant services for its own population. However, if live donation is to be encouraged, donors must be reassured that their health is no less important than that of the recipient. In 2008, a summit meeting was held in Istanbul to address the problems of organ trafficking in the context of a global shortage of donors; a key feature of the resultant Istanbul Declaration is the emphasis on follow-up for organ donors (Kidney Int. 74, 854-859 [2008]). The provision of full life insurance, complete coverage of medical expenses and financial compensation for loss of income should, therefore, be mandatory for any live donor. In the case that the donor's remaining kidney fails, donor patients should preferentially receive a kidney should they require a transplant.

The therapeutic potential of deceased organ donation also needs to be maximized by increasing the number of deceased donors and through the selective use of kidneys from expanded-criteria donors. The implementation of an informed, opt-out approach, in which all citizens are assumed to be organ donors unless they officially stipulate their refusal, is vital. The implementation of educational measures to prevent the development of kidney failure is also important. The high prevalence of renal failure in developed countries is largely a result of the increasing incidence of diabetes, obesity and hypertension. Public health initiatives are needed to reduce the prevalence of these conditions.

Until the disparity between the number of required organs and organ availability is reduced, the demand for donor organs will remain high and patients awaiting a transplant will desperately seek methods to prolong their life. Although the regulated financial-incentives model for live organ donation has some merit, the existence of such a system will inevitably exploit the poor who have little access to health care if their remaining kidney fails. It is essential that the health of the donor is considered to be as important as that of the recipient. The benefits of renal transplantation must outweigh the associated risks to both donor and recipient.

doi:10.1038/nrneph.2009.172

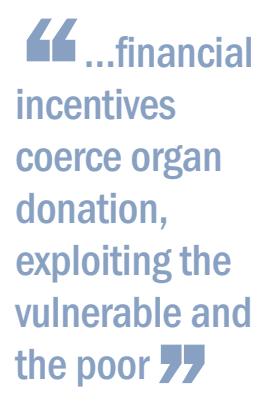

Susan J. Allison is the Editor of Nature Reviews Nephrology.

Competing interests The author declares no competing interests. 